\title{
3D heat diffusion modeling in defected multilayered media for IRT applications in building elements
}

\author{
by C. Serra*, A. Tadeu*** and N. Simões ${ }^{*, * *}$ \\ *ITeCons - Institute for Research and Technological Development for Construction, Energy, Environment and \\ Sustainability, Rua Pedro Hispano s/ n., 3030-289 Coimbra, Portugal, cserra@itecons.uc.pt \\ ${ }^{* *}$ ADAI - LAETA, Department of Civil Engineering, University of Coimbra, Pólo II, Rua Luís Reis Santos, \\ 3030-788 Coimbra, Portugal
}

\begin{abstract}
This paper presents a numerical model to simulate heat transfer in layered media looking to contribute to the interpretation of thermographic results obtained in building envelope inspections. 3D heat diffusion by conduction in defected multilayered media is modeled using a formulation of the Boundary Element Method (BEM) in the frequency domain incorporating Green's functions. The defect is a 3D null thickness inclusion. A TBEM formulation is used in order to handle the null thickness. The present paper presents a brief selection of preliminary results obtained.
\end{abstract}

\section{Introduction}

Infrared Thermography (IRT) has been established as a prominent non-destructive testing technique used in many sectors and for various applications [1]. For a long time, IRT in buildings has been mainly used to assess the quality of a building's envelope [2]. However, combining advanced thermal data processing techniques with experimental test results can lead to the characterization of defects hidden within building elements [3]. In particular, analyzing phase contrast images has been revealed to be more advantageous, when compared with using raw thermal contrast images $[4,5]$. Therefore, the adequate interpretation of IRT data demands for the development of suitable heat transfer modeling tools.

Aiming to contribute to the interpretation of IRT results obtained in building envelope inspection studies, this paper presents a numerical model used to simulate heat transfer in layered media using the 3D Boundary Element Method (BEM) formulated in the frequency domain and incorporating 3D Green's functions for multilayered media.

The problem is formulated in the frequency domain by applying a Fourier transform to the time domain diffusion equation and is based on the analytical solutions presented and validated by Tadeu $[6,7]$ for multilayered systems. In the present study, the analytical expressions are incorporated into a BEM formulation to model heat conduction in a layered medium containing a 3D defect, without requiring the discretization of the plane interfaces.

Compared with other modelling techniques, the BEM automatically satisfies far field conditions and therefore only requires the discretization of the inclusion's boundaries [8]. Even though its major drawback is that it requires the knowledge of fundamental solutions, the BEM is still considered to be one of the best tools for modelling homogeneous unbounded and semi-infinite media. In order to handle the thin defect, the BEM formulated in the frequency domain is written in terms of normal-derivative integral equations (TBEM) and known analytical solutions are used to solve the hypersingular integrals that appear in the TBEM formulation.

In this paper, the problem is defined and the TBEM formulation incorporating Green's functions for modelling layered media containing 3D defects is presented. The analytical solutions are formulated by adding the heat source terms equal to those in an unbounded space with the surface terms required to satisfy the boundary conditions at the interfaces between the layers. Total heat field is found by adding the heat source terms equal to those in an unbounded space with the surface terms generated at each interface in order to ensure the continuity of temperature and heat flux at each interface. The proposed formulation is verified using a previously 3D TBEM model [9] used to simulate the heat diffusion in the presence of thin defects embedded in an unbounded medium.

\section{Methodology}

\subsection{Problem definition}

In this work, a numerical model based on the 3D Boundary Element Method (BEM) incorporating 3D Green's functions for multilayered media is proposed to model heat diffusion by conduction in defected layered media. Consider a system of $m$ parallel layers (infinite in the $x$ and $z$ directions) bounded by a semi-infinite medium at the bottom (medium $m+1)$ and at the top (medium 0). Each layer may have different thickness and different thermal properties (thermal diffusivity $K_{j}$, thermal conductivity $\lambda_{j}$ density $\rho_{j}$ and specific heat capacity $c_{j}$ ). The defect is a 3D null thickness inclusion whose size, shape and position may vary. 


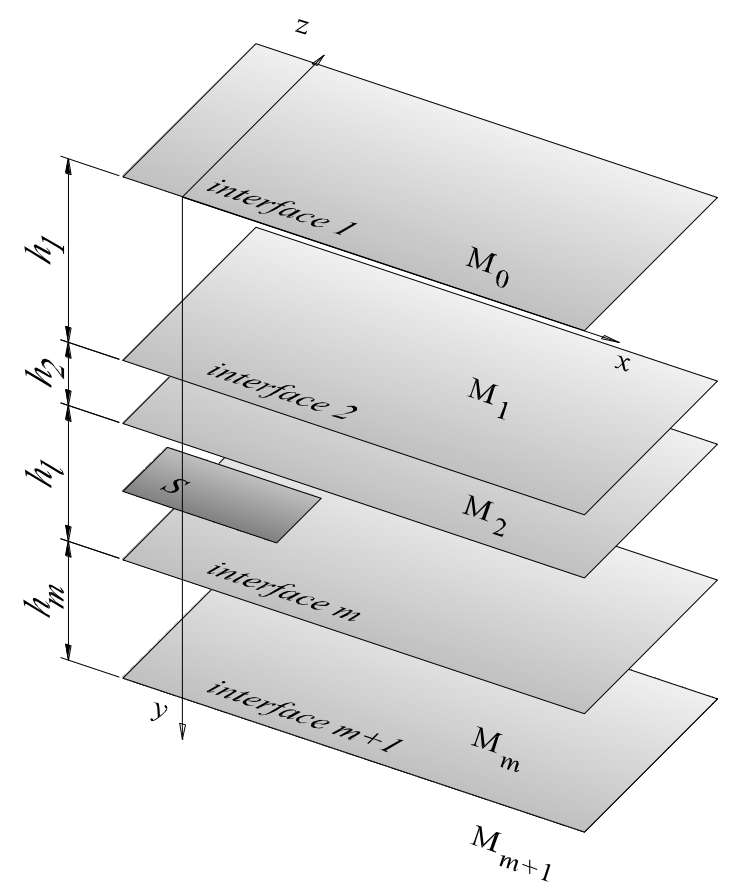

Fig. 1. Geometry of the problem of a multilayered system containing a $3 D$ thin defect.

\subsection{D heat diffusion in the multilayer system}

In the frequency domain, 3D heat diffusion in a homogenous isotropic solid unbounded medium is obtained by applying the Fourier transform to the heat diffusion equation, resulting in the following expression:

$$
\left(\frac{\partial^{2}}{\partial x^{2}}+\frac{\partial^{2}}{\partial y^{2}}+\frac{\partial^{2}}{\partial z^{2}}+\left(\sqrt{-\frac{\mathrm{i} \omega}{K}}\right)^{2}\right) \hat{T}(\omega, x, y, z)=0
$$

in which $\hat{T}(\omega, x, y, z)$ is temperature in the frequency domain, $\omega$ is angular frequency, $\mathrm{i}=\sqrt{-1}$, and $K$ is thermal diffusivity given by $K=\lambda /(\rho c)$, where $\lambda$ is thermal conductivity, $\rho$ is density and $c$ is specific heat capacity of the medium. Considering a 3D heat source placed at $\left(x_{s}, y_{s}, z_{s}\right)$ given by $p(x, y, z, t)=P \delta\left(x-x_{s}\right) \delta\left(y-y_{s}\right) \delta\left(z-z_{s}\right) e^{\mathrm{i} \omega t}$, where $\delta\left(x-x_{s}\right), \delta\left(y-y_{s}\right)$ and $\delta\left(z-z_{s}\right)$ are Dirac's delta functions and $P$ is heat source amplitude, the heat source response can be given by:

$$
\hat{T}_{i n c}(\omega, x, y, z)=\frac{P e^{-\mathrm{i} k r_{s}}}{2 \lambda r_{s}}
$$

in which $k=\sqrt{-\mathrm{i} \omega / K}$ and $r_{s}=\sqrt{\left(x-x_{s}\right)^{2}+\left(y-y_{s}\right)^{2}+\left(z-z_{s}\right)^{2}}$.

In a multilayered system, the presence of a 3D heat source creates a temperature field that is defined by the direct field incident within the layer where the source is placed and by the field created by the presence of the multilayer interfaces. The computation of the generated heat field requires the transformation of the direct incident field following the procedure proposed by Sommerfeld [10],[11]. This leads to a Bessel integral that can be expressed by the following sum (assuming the existence of virtual heat sources equally spaced by $L_{y}$ along the $y$ axis):

$$
\hat{T}_{i n c}(\omega, x, y, z)=\frac{-\mathrm{i} \pi}{L_{y} \lambda} \sum_{n=1}^{N} \mathrm{~J}_{0}\left(k_{n} \bar{r}_{s}\right) \frac{k_{n}}{v_{n}} e^{-\mathrm{i} \mathrm{v}_{n}\left|y-y_{s}\right|},
$$

in which $\mathrm{J}_{\mathrm{n}}\left(\mathrm{)}\right.$ are Bessel functions of order $\mathrm{n}, \bar{r}_{s}=\sqrt{\left(x-x_{s}\right)^{2}+\left(z-z_{s}\right)^{2}}, v_{n}=\sqrt{-\mathrm{i} \omega / K-k_{n}^{2}}, k_{n}=2 \pi n / L_{y}$ and $L_{y}$ must be big enough so as to prevent spatial contamination from the virtual sources.

The field generated by the presence of all different interfaces can be computed assuming the existence of a set of surface terms, whose amplitude is defined so as to ensure the continuity of heat fluxes and temperatures. Hence, the resulting heat field is the sum of the source term and the surface terms generated at each interface. It can be 
demonstrated that the heat field generated by those surface terms, within any layer $j$, other than medium 0 or medium $m+1$, is given by:

$$
\hat{T}(\omega, x, y, z)=\frac{-i \pi P}{L_{y} \lambda_{j}} \sum_{n=1}^{N} \mathrm{~J}_{0}\left(k_{n} \bar{r}_{s}\right) \frac{k_{n}}{v_{n j}} A_{n j}^{1},
$$

in which $k_{j}=\sqrt{-\mathrm{i} \omega / K_{j}}, v_{n j}=\sqrt{-\mathrm{i} \omega / K_{j}-k_{n}^{2}}$ with $\operatorname{Im}\left(v_{n j}\right) \leq 0, A_{n j}^{1}=A_{n j}^{t} e^{-\mathrm{i} v_{n j}\left|y-\sum_{l=1}^{j-1} h_{l}\right|}+A_{n j}^{b} e^{-\mathrm{i} v_{n j}\left|y-\sum_{l=1}^{j} h_{l}\right|}$, and $A_{n j}^{t}$ and $A_{n j}^{b}$ are unknown potential amplitudes at the top and bottom of each layer $j$ and $h_{l}$ is the thickness of layer $l$. The heat field in medium 0 , medium $\mathrm{m}+1$ can be deduced accordingly (not shown in this paper). In the layer containing the heat source the final heat field is given by adding the direct incident heat field.

\subsection{D heat diffusion in the multilayer system containing a thin defect}

The heat field created by inclusions within the layered system can be modelled using a boundary element technique that only requires the discretization of the inclusion. When thin defects are present, the conventional direct BEM may degenerate and become inaccurate. This is frequently solved by using the normal-derivative integral equations formulation (TBEM). This equation can be derived by applying the gradient operator to the boundary integral equation assuming the existence of dipole heat sources. The solution to the problem is obtained by solving the normal derivative integral equation, which requires only the discretization of the surface of the defect $S$ into $N$ planar boundary elements with one nodal point at the center of each element. When boundary $S$ is loaded with dipoles the integral equation is expressed by:

$$
a T\left(\omega, x_{0}, y_{0}, z_{0}\right)=-\int_{S} \bar{H}\left(\omega, x, y, z, x_{0}, y_{0}, z_{0}, \mathbf{n}_{n 1}, \mathbf{n}_{n 2}\right) T(\omega, x, y, z) d s+\bar{T}_{i n c}\left(\omega, x_{s}, y_{s}, z_{s}, x_{0}, y_{0}, z_{0}, \mathbf{n}_{n 2}\right),
$$

in which $\mathbf{n}_{n 1}$ represents the unit outward normal to the boundary $S$ at point $(x, y, z), \mathbf{n}_{n 2}$ represents the unit outward normal to the boundary $S$ at the collocation points $\left(x_{0}, y_{0}, z_{0}\right)$ and $a$ is null for planar piecewise boundary elements.

When the source is in the same layer as the defect the incident heat field $\bar{T}_{i n c}$ is given by the sum of the field generated by the source and the reflections that occur at each interface between layers:

$$
\begin{aligned}
& \bar{T}_{i n c}\left(\omega, x, y, z, x_{s}, y_{s}, z_{s}, \mathbf{n}_{n 2}\right)=\frac{P e^{-\mathrm{i} k_{j} r_{s}}\left(-\mathrm{i} k_{j} r_{s}-1\right)}{2 \lambda_{j} r_{s}^{2}}\left(\frac{\partial r_{s}}{\partial x} \frac{\partial x}{\partial \mathbf{n}_{n 2}}+\frac{\partial r_{s}}{\partial y} \frac{\partial y}{\partial \mathbf{n}_{n 2}}+\frac{\partial r_{s}}{\partial z} \frac{\partial z}{\partial \mathbf{n}_{n 2}}\right) \\
& +\frac{-i \pi P}{L_{y} \lambda_{j}} \sum_{n=1}^{N}\left[\left(-k_{n}\right) \mathrm{J}_{1}\left(k_{n} \bar{r}_{s}\right)\left(\frac{\partial \bar{r}_{s}}{\partial x} \frac{\partial x}{\partial \mathbf{n}_{n 2}}+\frac{\partial \bar{r}_{s}}{\partial z} \frac{\partial z}{\partial \mathbf{n}_{n 2}}\right) \frac{k_{n}}{v_{n j}} A_{n j}^{1}+\mathrm{J}_{0}\left(k_{n} \bar{r}_{s}\right) \frac{k_{n}}{v_{n j}}\left(-\mathrm{i} v_{n j}\right) A_{n j}^{2} \frac{\partial y}{\partial \mathbf{n}_{n 2}}\right]^{\prime} \\
& \text { in which } A_{n j}^{2}=A_{n j}^{t} e^{-\mathrm{i} v_{n j}\left|y-\sum_{l=1}^{j-1} h_{l}\right|}-A_{n j}^{b} e^{-\mathrm{i} v_{n j}\left|y-\sum_{l=1}^{j} h_{l}\right|} .
\end{aligned}
$$
for temperature:

The required Green's fundamental solutions $\bar{H}$ are derived manipulating mathematically the Green's functions

$$
G\left(\omega, x, y, z, x_{0}, y_{0}, z_{0}\right)=\frac{e^{-\mathrm{i} k_{j} r_{0}}}{4 \pi \lambda_{j} r_{s}}+\frac{-\mathrm{i} P}{2 L_{y} \lambda_{j}} \sum_{n=1}^{N} \mathrm{~J}_{0}\left(k_{n} \bar{r}_{0}\right) \frac{k_{n}}{v_{n j}} A_{n j}^{1}
$$

For sake of brevity those derivations are not presented in this paper. The solution to the problem is obtained by solving the normal derivative integral equation (5), after discretization of the defect surface $S$ with $N$ boundary surface elements. This leads to a system of $N \times N$ equations that require the integration $\bar{H}^{k l}=\int_{B_{l}} \bar{H}\left(\omega, x_{l}, y_{l}, z_{l}, \mathbf{n}_{n 1}, \mathbf{n}_{n 2}, x_{k}, y_{k}, z_{k}\right) d B_{l}$ in which $l$ is the element being integrated, $k$ is the loaded element, and $B_{l}$ is the surface of boundary element $l$. When the element to be integrated is not the loaded element the integrations are evaluated using a Gaussian quadrature scheme. For the loaded element the integrands exhibit a singularity (hypersingular element). Such singularities may be solved analytically using known solutions for cylindrical elements [12].

\subsection{Verification}

The verification of the proposed algorithm was performed using a 3D TBEM formulation for modeling 3D heat diffusion in the vicinity of a 3D thin inclusion [9] embedded in an unbounded medium which has been previously validated against known analytical solutions. 
In order to enable the comparison with the 3D TBEM model, two separate systems were modeled: half-space with prescribed null normal heat fluxes and null temperatures along the surface. Using the proposed 3D TBEM multilayered model, heat diffusion was modeled in a medium which consists of layers with properties equal to those of the infinite unbounded medium simulated using the previous 3D TBEM model. At the top, null normal heat fluxes are achieved by imposing an extremely low conductivity while null temperatures are enforced by imposing an extremely high conductivity. In the 3D TBEM model for an unbounded medium, null heat fluxes are achieved by adding the heat field generated by the real source and the real inclusion to that which is produced by a virtual source and a virtual inclusion (which act as a mirror). Similarly, null temperature is accomplished by using a virtual source with negative amplitude in order to guarantee the required boundary conditions.

To perform the validation, a medium with a conductivity of $\lambda=1.4 \mathrm{~W} /\left(\mathrm{m} .{ }^{\circ} \mathrm{C}\right)$, heat capacity of $c=880 \mathrm{~J} /\left(\mathrm{kg} .{ }^{\circ} \mathrm{C}\right)$ and density of $\rho=2300 \mathrm{~kg} / \mathrm{m}^{3}$ subject to a 3D source placed at $x_{s}=0.03 \mathrm{~m}, y_{s}=0.04 \mathrm{~m}$ and $z_{s}=0.03 \mathrm{~m}$ was considered. The defect consists of a $0.05 \times 0.05 \mathrm{~m}^{2}$ thin inclusion placed parallel to the $x z$ plane at $y=0.05 \mathrm{~m}$. The geometry of the system modeled for this validation is shown in Figure 2. Computations were performed over three grids of receivers (illustrated in Figure 3). One grid is placed parallel to plane $x y$ at $z=0$ and the other two grids (G2 and G3) are placed parallel to the plane inclusion (at $y=0.025 \mathrm{~m}$ and at $y=0.075$ ). The receivers are spaced at equal intervals of $0.0020 \mathrm{~m}$ in the $x$ and $z$ directions and $0.0013 \mathrm{~m}$ in the $y$ axis direction.

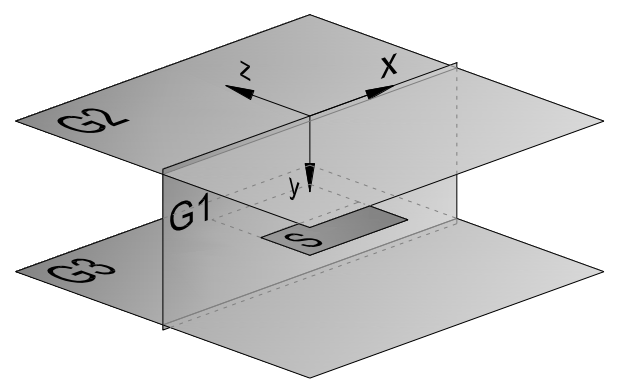

Fig. 2. 3D view of the geometry of the system modelled.

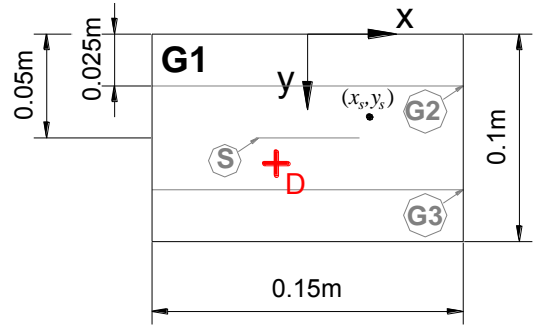

a)

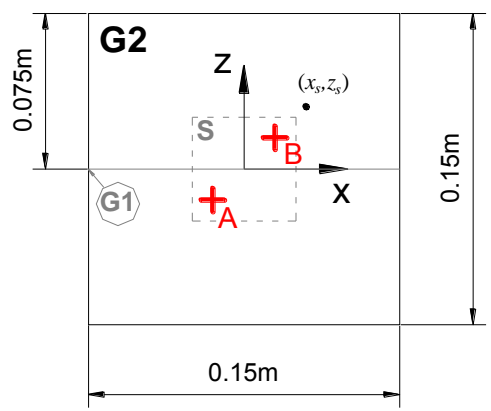

b)

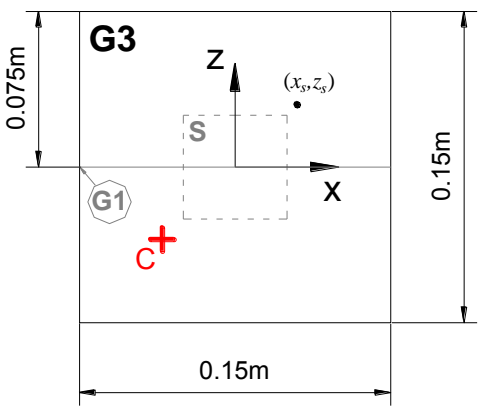

c)

Fig. 3. $2 D$ view of the geometry of the system modelled: a) $x y$ plane and placement of receiver $D$; $b) x z$ plane at $y=0.025 \mathrm{~m}$ and placement of receivers $A$ and $B$; c) $x z$ plane at $y=0.075 \mathrm{~m}$ and placement of receiver $C$.

The results obtained for a range of frequencies [ 0 to $6.35 \times 10^{-5} \mathrm{~Hz}$ ] at receivers $A, B, C$ and $D$ are shown in the following graphs. Figure 4 compares the temperature response computed using the proposed 3D TBEM multilayered and the previous 3D TBEM model for the case of null heat flux. Figure 5 compares the results obtained using the multilayered and the 3D TBEM model for the case of null temperature. The analyses of these figures allow the verification of the correctness of the proposed solutions. 


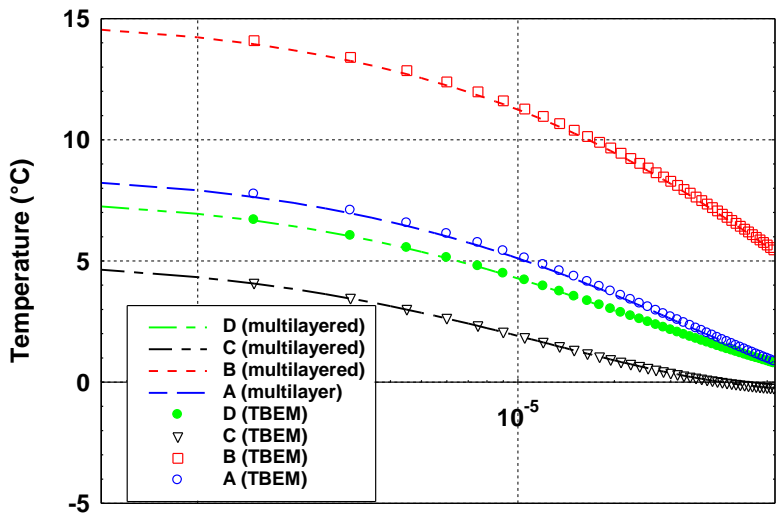

$f(\mathrm{~Hz})$

a)

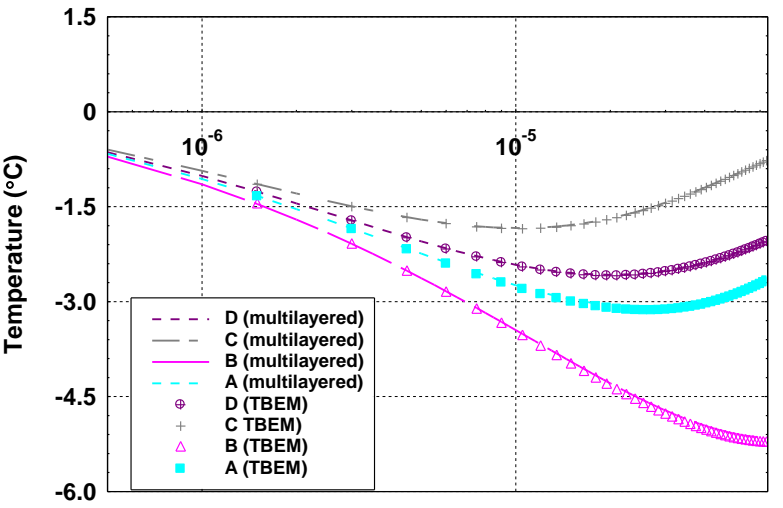

$f(\mathrm{~Hz})$

Fig. 4. Responses of the multilayered model and the TBEM model for the case of null heat flux recorded in receivers $A, B, C$ and $D:$ a) real part; $b$ ) imaginary part.

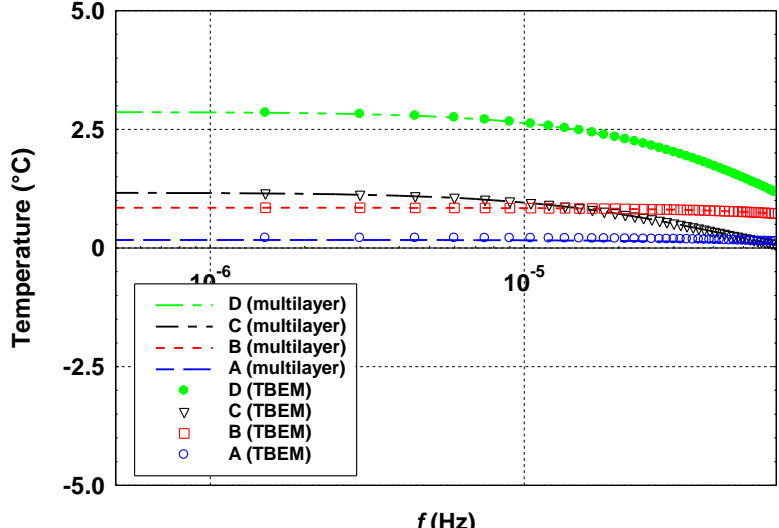

a)

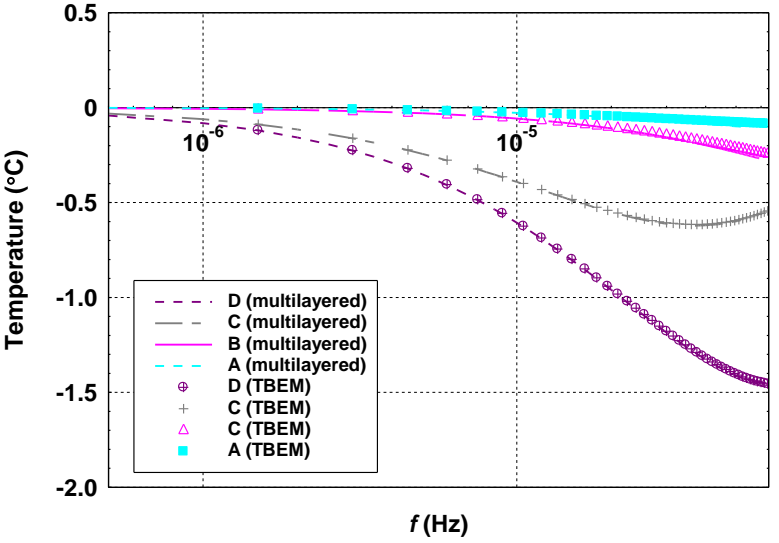

b)

Fig. 5. Responses of the multilayered model and the TBEM model for the case of null temperature recorded in receivers $A, B, C$ and $D:$ a) real part; $b$ ) imaginary part.

Figure 6 illustrates the responses along the defined grid of receivers G1, G2 and G3 for null frequency when null heat fluxes are prescribed along the surface. The responses obtained along the grid of receivers $\mathrm{G} 1$ allow the clear verification of the perturbation caused by the thin defect. It also shown that, as expected, the contour lines are perpendicular to the half space surface.

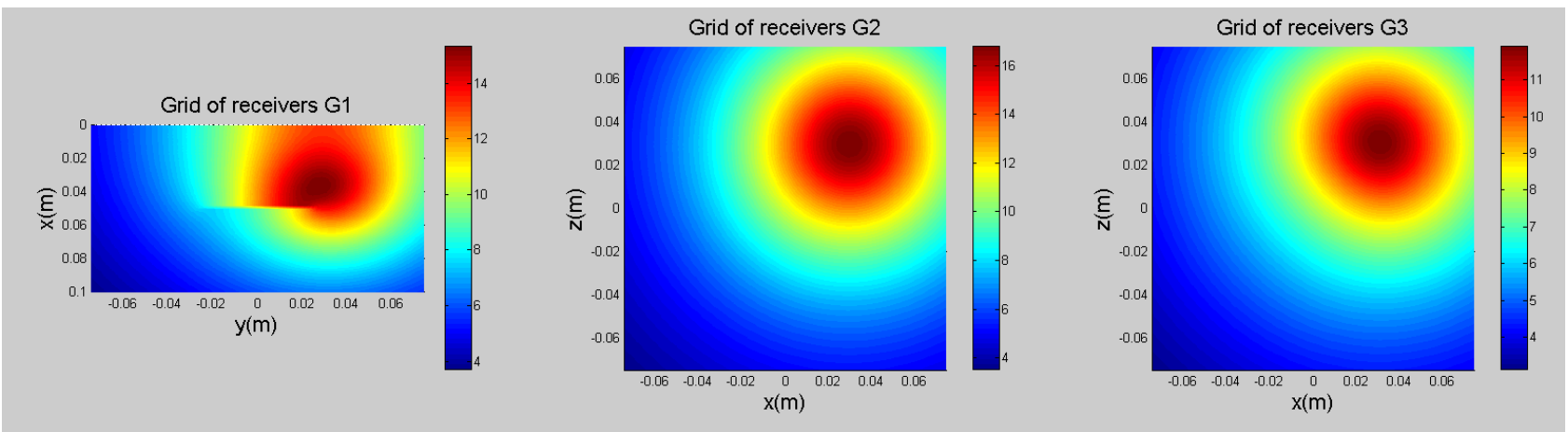

Fig. 6. Responses along the defined grid of receivers G1, G2 and G3 for null frequency when null heat fluxes are prescribed along the half space surface. 


\section{Conclusions}

This paper presents an extension to the work developed previously by the authors, where applications using a 3D TBEM formulated in the frequency domain were used to model transient heat transfer by diffusion in unbounded solid media with defects [9],[13] to multilayered media containing 3D thin defects. This paper presents some of the preliminary results, namely the verification of the proposed model. This model will be used in a number of numerical applications including defects with varying size, shape and position. These applications are expected to be useful for assessing the influence that the presence of a defect with varying characteristics has on the heat diffusion response and, in particular, on phase contrast results. Thermal wave phase results will be compared with those obtained when the defect is absent, so as to study the effect of the presence of the defect.

\section{AKNOWLEDGEMENTS}

The research work has been framed under the Initiative Energy for Sustainability of the University of Coimbra. This work has also been supported was supported in part by QREN - Compete under the research project FCOMP-01-0202FEDER-033938 and by the doctoral FCT grant SFRH/BD/91686/2012.

\section{REFERENCES}

[1] Maldague X., "Theory and Practice of Infrared Technology for Non-destructive Testing", John Wiley \& Sons, 2001.

[2] Balaras C. A., Argiriou A. A., Infrared thermography for building diagnostics, Energy and Buildings, Vol. 34 (2002), pp. 171-183.

[3] Grinzato E., Vavilov V., Kauppinen T., Quantitative infrared thermography in buildings, Energy and Buildings, Vol. 29 (1998), pp. 1-9.

[4] Maldague X., Marinetti S., Pulse phase infrared thermography, Applied Physics, Vol. 79, no.5 (1996), pp. 26942698.

[5] Arndt R.W., Square pulse thermography in frequency domain as adaptation of pulsed phase thermography for qualitative and quantitative applications in cultural heritage and civil engineering, Infrared Physics \& Technology, Vol. 53, no. 4 (2010), pp. 246-253.

[6] Tadeu A., António J., Simões N., 2.5D Green's functions in the frequency domain for heat conduction problems in unbounded, half-space, slab and layered media, Computer Modeling in Engineering and Sciences - CMES, Vol. 6, no 1 (2004), pp. 43-58.

[7] Tadeu A., Simões N., Three-dimensional fundamental solutions for transient heat transfer by conduction in an unbounded medium, half-space, slab and layered media, Engineering Analysis with Boundary Elements, Vol. 30 , no. 5 (2006), pp. 338-349.

[8] Brebbia C.A., Telles J.C., Wrobel L.C., "Boundary Elements Techniques: Theory and Applications in Engineering", Springer-Verlag, Berlin-New York, 1984.

[9] Serra C., Tadeu A., Prata J., Simões N., Application of 3D heat diffusion to detect embedded 3D empty cracks, Applied Thermal Engineering, Vol. 61, no. 2 (2013), pp. 596-605.

[10] Sommerfeld A. "Mechanics of deformable bodies", New York: Academic Press, Inc., 1950.

[11] Ewing W.M., Jardetzky W.S., Press F. "Elastic Waves in Layered Media”, McGraw-Hill Book Company, 1957.

[12] Tadeu A., Prata J., Simões N., Closed form integration of singular and hypersingular integrals in 3D BEM formulations for heat conduction, Mathematical Problems in Engineering (2012), Article ID 647038, doi:10,1155/2012/647038.

[13] Serra C., Tadeu A., Simões N., 3D heat diffusion simulation using 3D and 1D heat sources - Temperature and phase contrast results for defect detection using IRT, Applied Mathematical Modelling, Vol. 40, no.2 (2016) pp.1576-1587. 